\title{
Ballistic Fracturing of Carbon Nanotubes
}

Sehmus Ozden ${ }^{1, *}$, Leonardo D. Machado ${ }^{2,3, t}$, ChandraSekhar Tiwary ${ }^{1, t}$, Pedro A. S. Autreto ${ }^{2,4}$,

Robert Vajtai ${ }^{1}$,Enrique V. Barreral ${ }^{1}$, Douglas S. Galvao ${ }^{2}$, Pulickel M. Ajayan ${ }^{1, *}$

${ }^{1}$ Department of Material Science and NanoEngineering, Rice University, Houston, Texas 77005,USA,

${ }^{2}$ Applied Physics Department, State University of Campinas, Campinas-SP, 13083-959, Brazil,

${ }^{3}$ Departamento de Física Teórica e Experimental, Universidade Federal do Rio Grande do Norte,

Natal-RN, 59072-970, Brazil

${ }^{4}$ Universidade Federal do ABC, Santo André-SP, 09210-580, Brazil

\section{Supplementary Information}

(a)

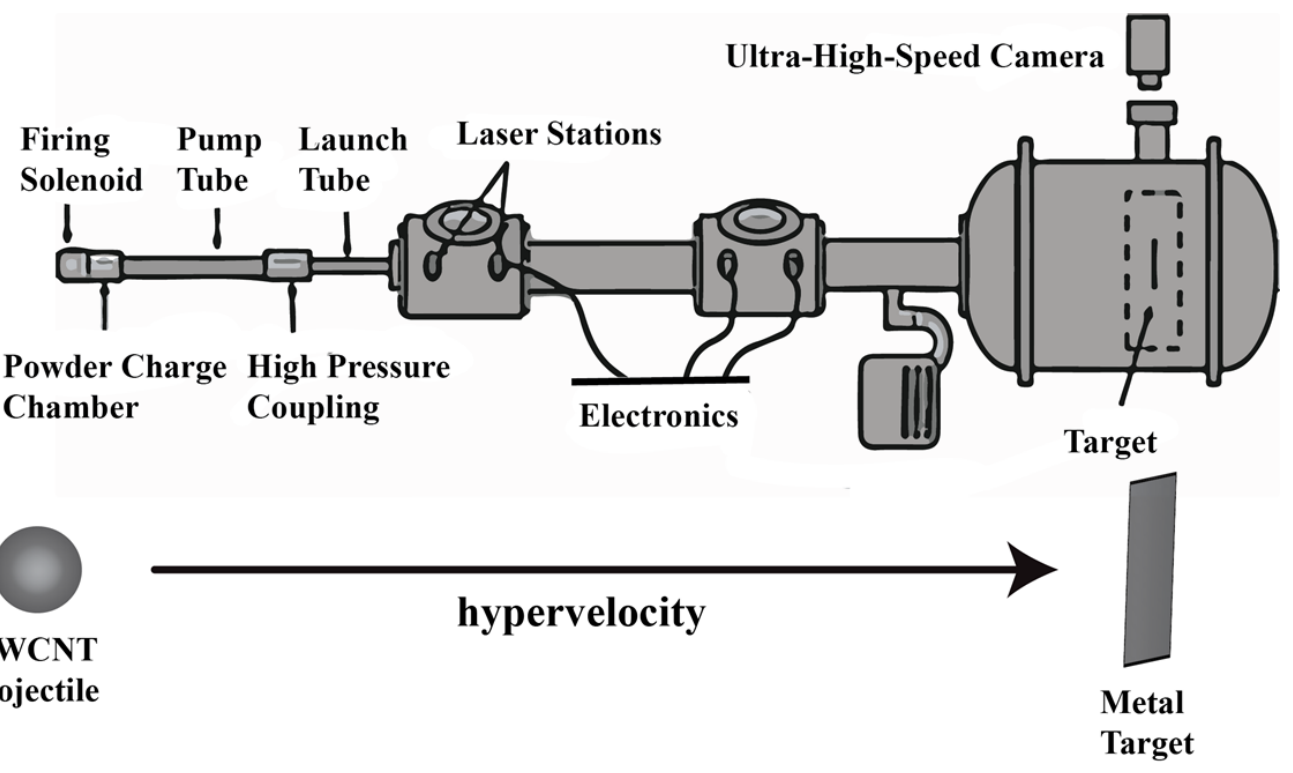

Figure S1. (a) Schematic of the specially designed two-stage light gas gun (LGG) at Rice University used for hypervelocity impact (HVI). The individual parts are marked in the figure, (b) Schematic figure of the target configuration for the HVI experiment of spherical CNTs. 

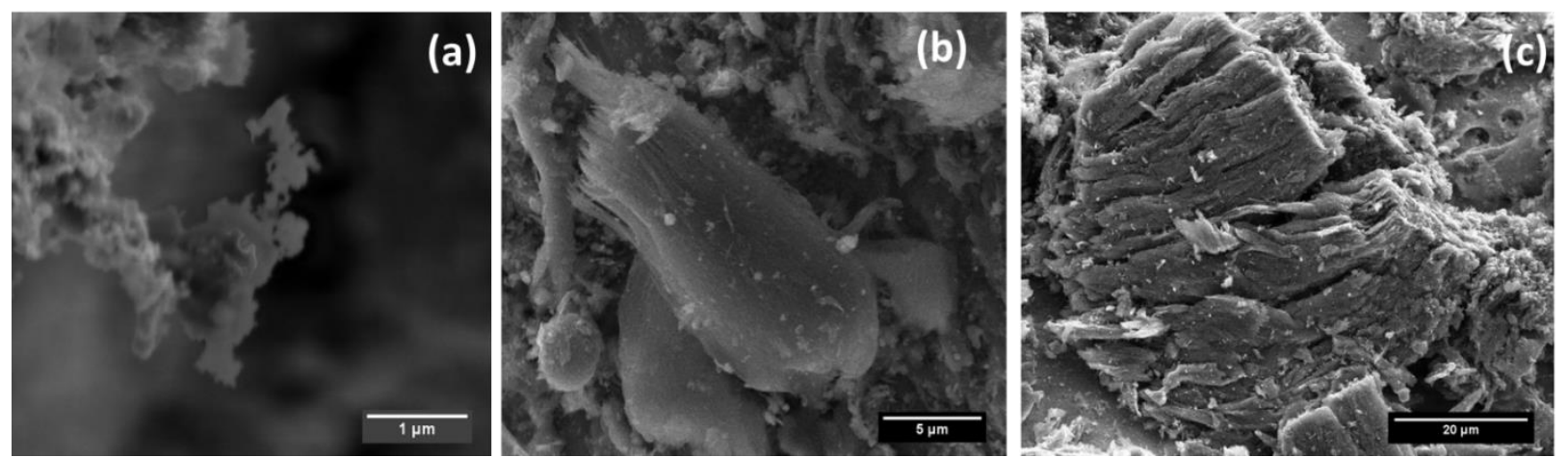

Figure S2. Scanning Electron Microscope (SEM) images of (a) hypervelocity impacts, (b) high velocity impact, and (c) low velocity impact.
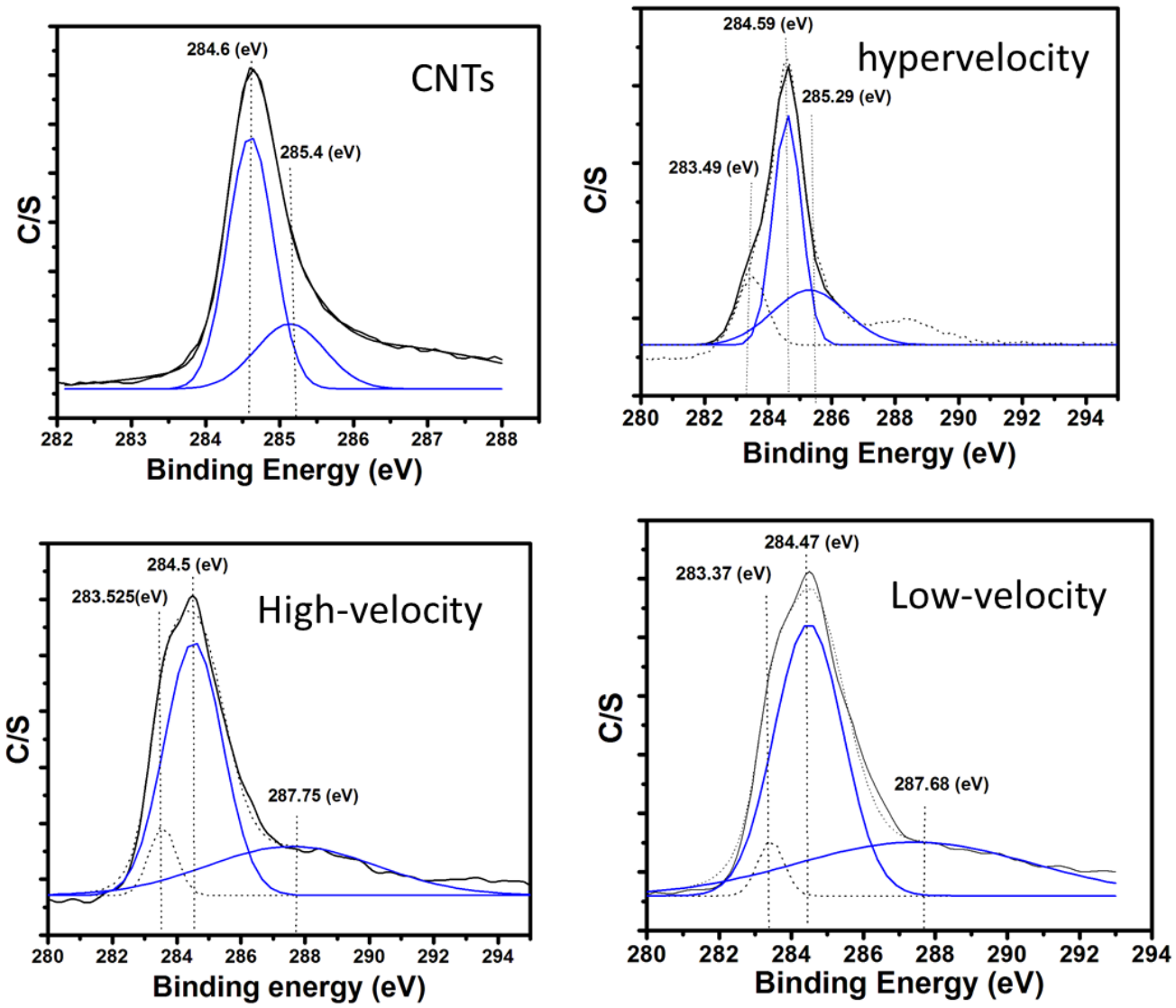

Figure S3. XPS characterization of (a) carbon nanotubes (CNTs), (b) hypervelocity impact, (c) high-velocity impact, (d) low-velocity impact 


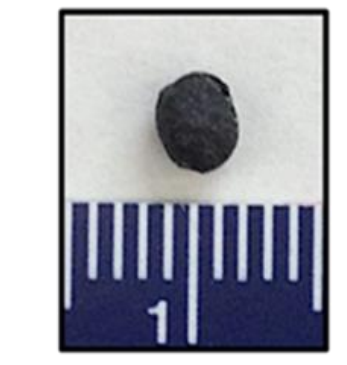

CNTs projectile before impact test

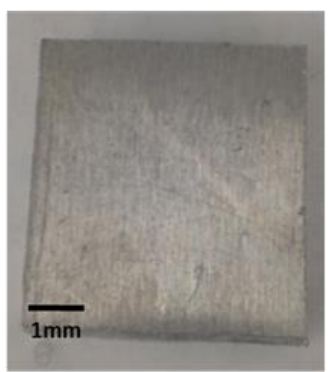

Al target before impact test

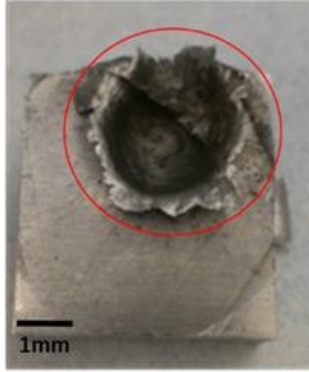

Al target After impact test

Figure S4. The size of the CNTs spherical ball is $\sim 2 \mathrm{~mm}$ before the impact test. The digital image of the target with impacted CNT spherical ball is shown. It clearly revels that the sphere retains its size and shape.

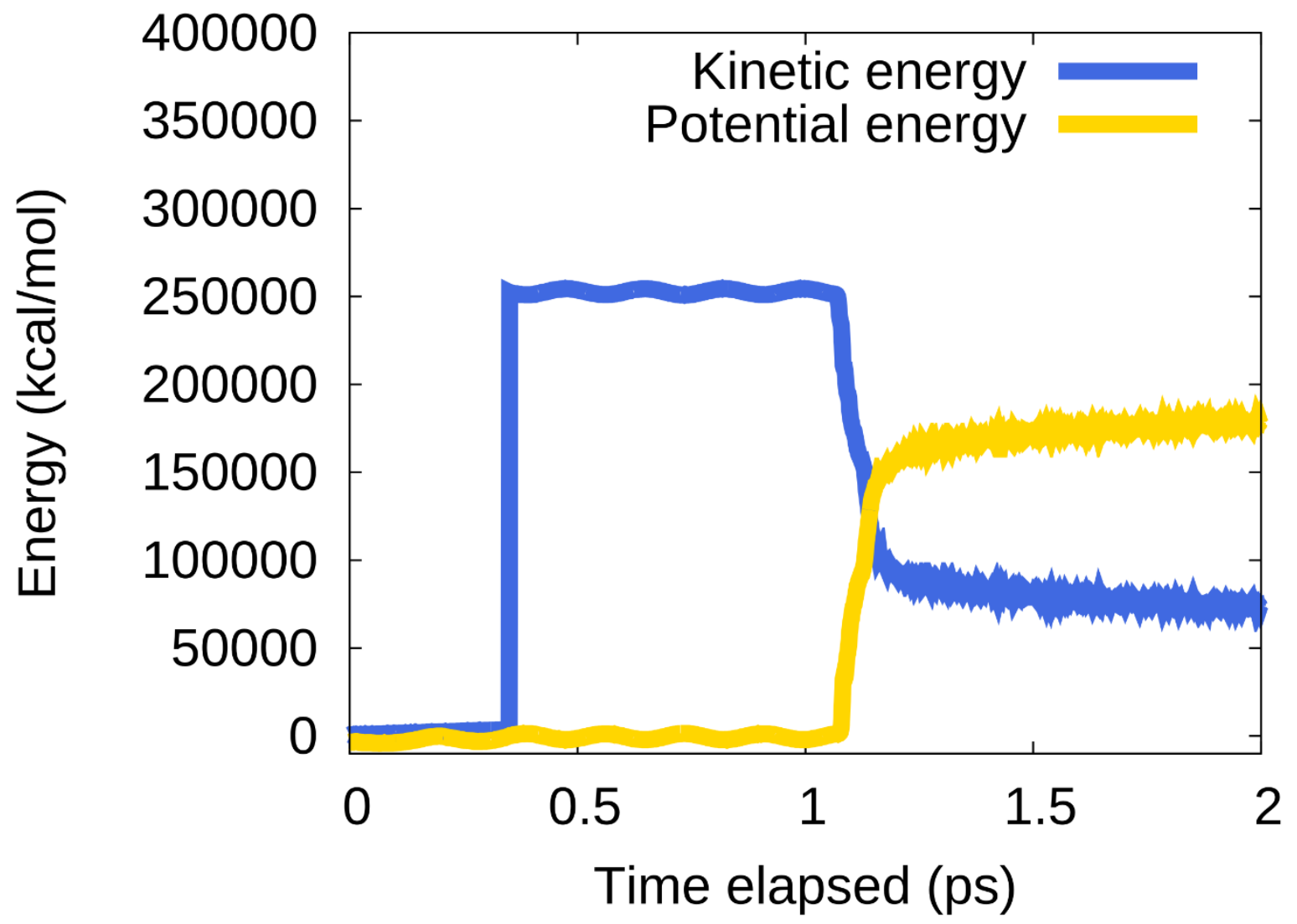

Figure S5: Energy profile for the lateral impact of a DWNT against a target at $6.5 \mathrm{~km} / \mathrm{s}$. Energy values are not normalized per atom; the initial potential energy was defined as zero. Since energy is constant in the simulations, a decrease in kinetic energy must correspond to an increase in potential energy. For such a high velocity impact, $27.7 \%$ of all bonds were broken. In such high 
velocity cases, most of the energy increase is due to broken bonds. The following rough calculation can aid in understanding this: the initial binding energy was $-757284 \mathrm{kcal} / \mathrm{mol}$. An estimative of the energy increase due to bond breakage can be found by multiplying the fraction of broken bonds by the total energy: $209768 \mathrm{kcal} / \mathrm{mol}$. The actual increase was lower than this, since bond order of the remaining bonds can increase to compensate for those that were broken. For lower velocities, the fraction of broken bonds is much lower, and structural deformation accounts for much of the energy increase.

\section{Molecular Dynamics simulation methods: additional information}

Finding broken bonds percentage: The first step of the algorithm consisted in finding the total number of bonds before impact. We used a geometrical criteria to determine whether two atoms were bonded: if the distance between them was less than $1.75 \AA$ we considered them bonded. Although typical equilibrium carbon-carbon bond length is less than $1.54 \AA$, we used a larger value because bond length oscillates during dynamics. For each atom we counted the number of neighbors, and added these numbers to obtain the total number of neighbors. We divided this number by 2 to obtain the initial number of bonds. We repeated this procedure after impact, to obtain the final number of bonds. We used this data to calculate the percentage of broken bonds.

Finding $\mathbf{s p}^{3}$ carbon percentage: We used the same geometrical criteria described above to determine whether two atoms are bonded. In the first step of the algorithm, we determined the number of neighbors for each atom. We considered that an atom had $\mathrm{sp}^{3}$ hybridization if it had four neighbors. By counting the number of atoms that satisfied this criteria, we obtained the total number of $\mathrm{sp}^{3}$ carbon. By dividing this amount by the total number of carbon atoms, we obtained the percentage. 


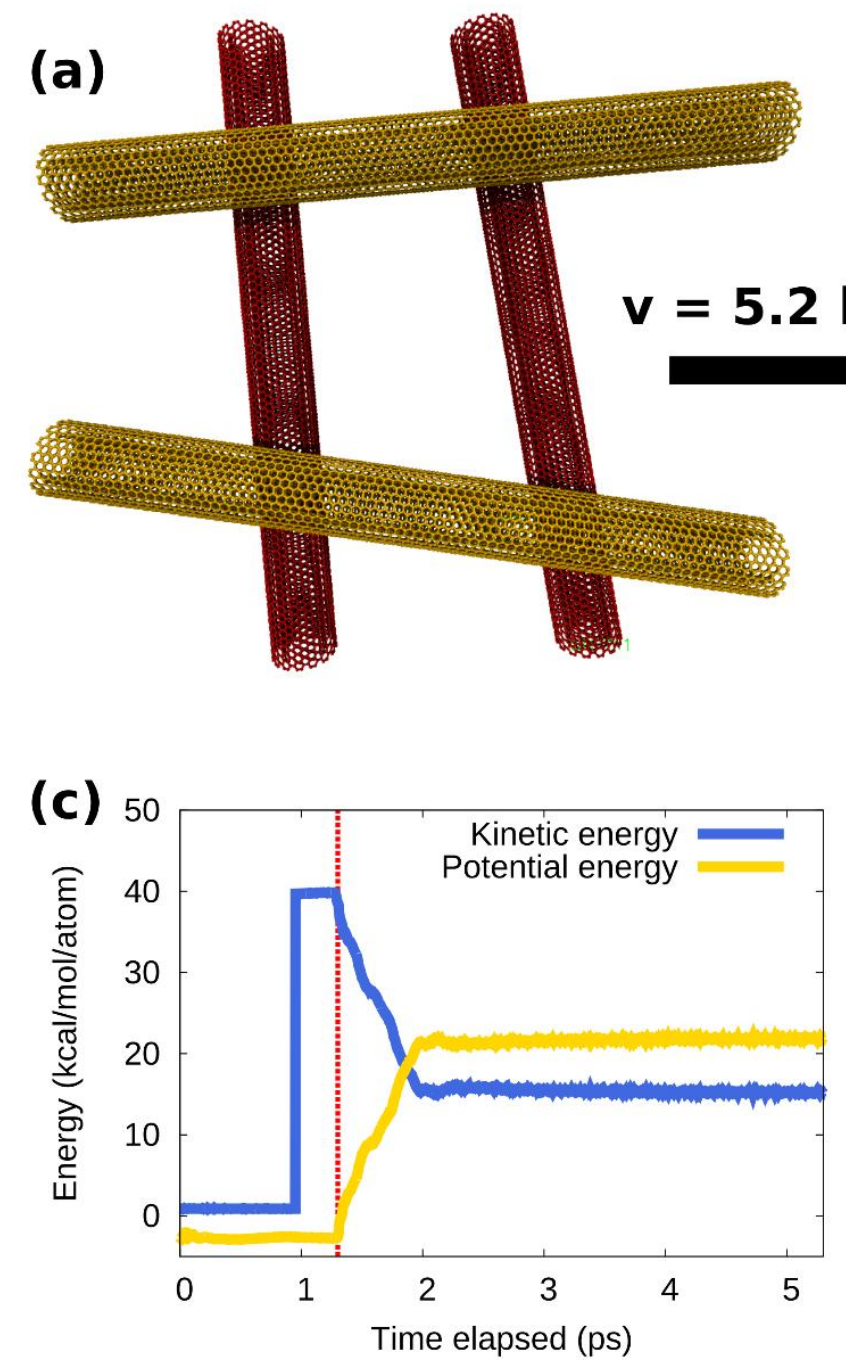

(b)
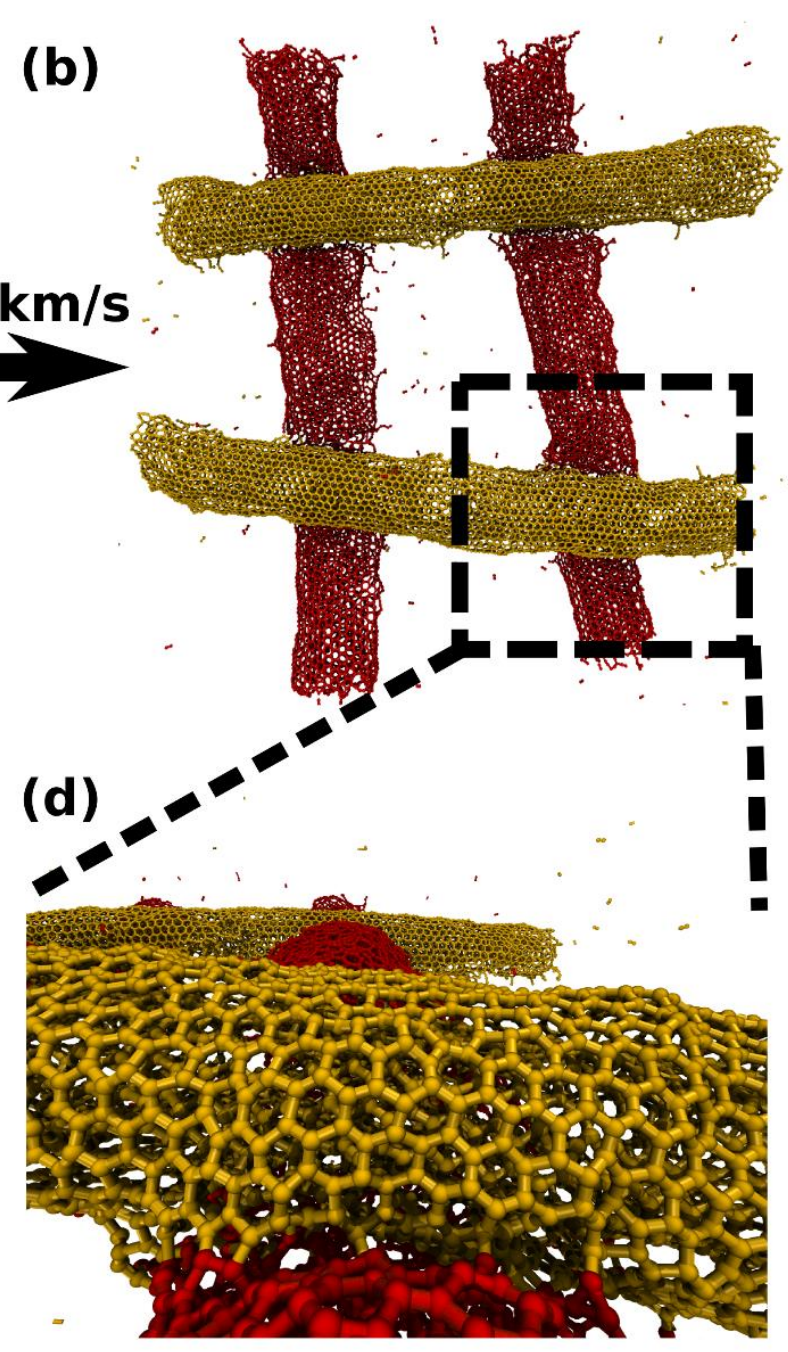

Figure S6: Simulation results in which a non-orthogonal array of CNTs collides with a solid target at a velocity of $5.2 \mathrm{~km} / \mathrm{s}$. (a-b) are, respectively, snapshots before and after impact. (c) Presents the evolution of the kinetic and potential energies during the simulation. (d) Presents a zoom-in of a CNT-CNT junction. Inter-nanotube covalent bonding can also be observed in this case. 
Defect-free

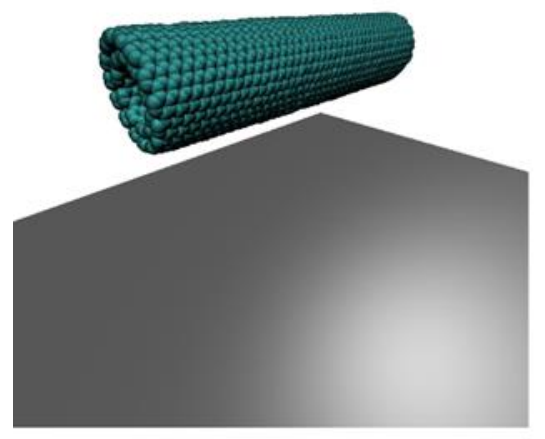

\section{Impact \\ $v=5.5 \mathrm{~km} / \mathrm{s}$}
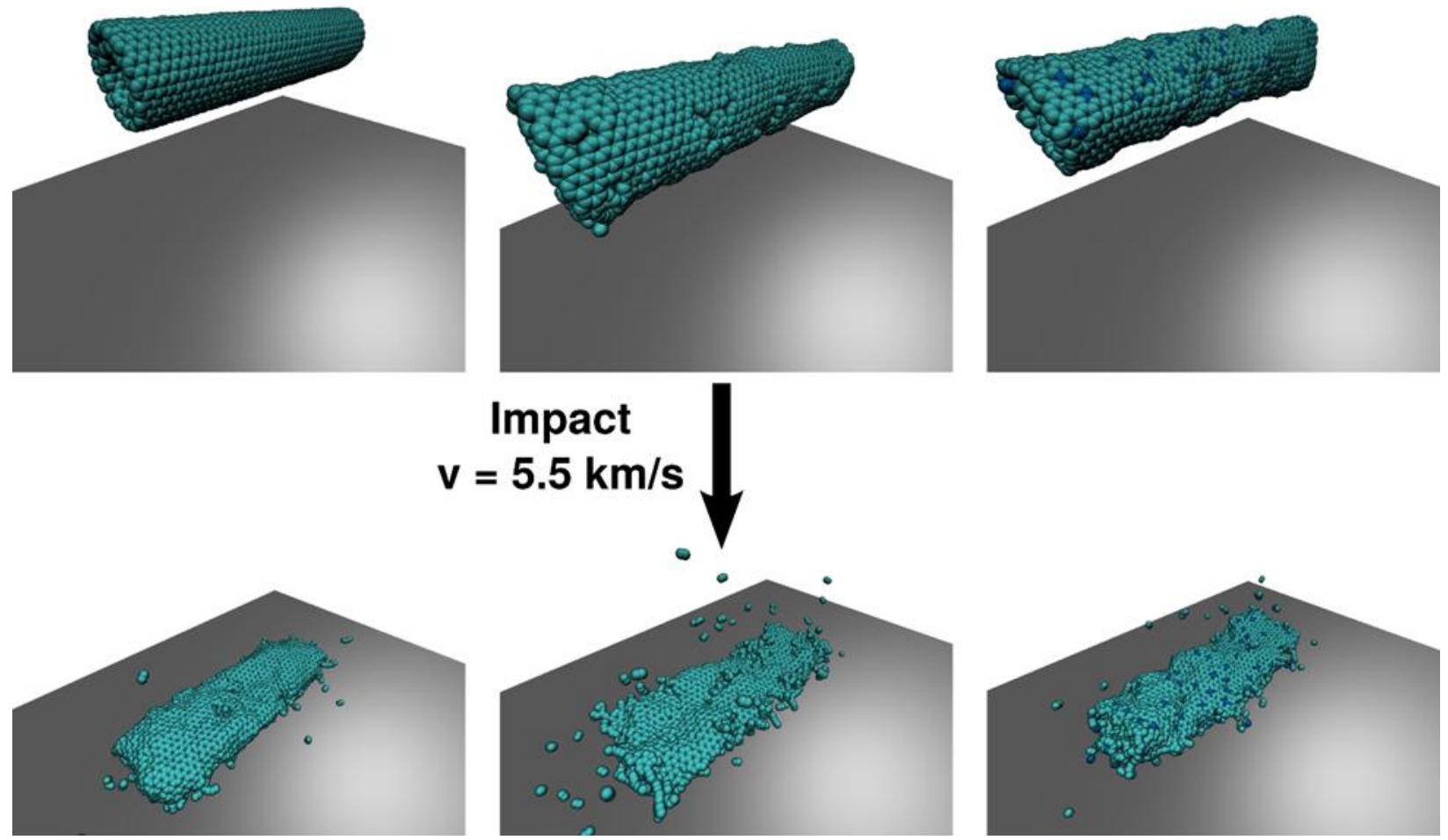

Figure S7: Results of HVI simulations, for nanotubes without defects, with vacancies (5\%) and with Stone-Wales defects (2.5\% of bonds rotated). Similar outcomes were observed regardless of presence and type of defect. See also movie S3. 